\title{
Multivariate quantum memory as controllable delayed multiport beamsplitter
}

\author{
A. N. Vetlugin, and I. V. Sokolov \\ St. Petersburg State University, SPbSU, \\ 7/9 Universitetskaya nab., \\ St. Petersburg, 199034 Russia
}

\begin{abstract}
The addressability of parallel spatially multimode quantum memory for light allows one to control independent collective spin waves within the same cold atomic ensemble. Generally speaking, there are transverse and longitudinal degrees of freedom of the memory that one can address by a proper choice of the pump (control) field spatial pattern. Here we concentrate on the mutual evolution and transformation of quantum states of the longitudinal modes of collective spin coherence in the cavity-based memory scheme. We assume that these modes are coherently controlled by the pump waves of the on-demand transverse profile, that is, by the superpositions of waves propagating in the directions, close to orthogonal to the cavity axis. By the write-in, this allows one to couple a time sequence of the incoming quantized signals to a given set of superpositions of orthogonal spin waves. By the readout, one can retrieve quantum states of the collective spin waves that are controllable superpositions of the initial ones and are on demand coupled to the output signal sequence. In general case, the memory is able to operate as a controllable delayed multi-port beamsplitter, capable of transformation of the delays, the durations and time shapes of signals in the sequence.

We elaborate the theory of such light-matter interface for the spatially multivariate cavity-based off-resonant Raman-type quantum memory. Since in order to speed up the manipulation of complex signals in multivariate memories it might be of interest to store relatively short light pulses of a given time shape, we also address some issues of the cavity-based memory operation beyond the bad cavity limit.
\end{abstract}

\section{INTRODUCTION}

The basic operations of quantum information protocols include creation, transformation and communication with non-classical states. In continuous variables quantum information one manipulates quadrature components of light, collective spin coherence, etc. The appropriate tool for these operations is provided by quantum memories [1-3].

Atomic ensembles and solid state systems with multiple degrees of freedom are used in order to extend the quantum memory capacity by applying the multimode in time, frequency or spatial domain protocols. The inclusion of the quantized light signal transverse degrees of freedom, as well as of non-collinear configurations of the interacting waves [4 7], allowed one to consider the quantum memory device as a quantum hologram.

In the spatially multimode memories the number of stored transverse modes is limited by diffraction or even by the geometric aperture of atomic ensemble in some configurations. There were demonstrated theoretically the offresonant QND quantum memory for images [4], and the spatially resolving off-resonant Raman-type memory [6]. The quantum volume hologram [7, 8] combines the Raman-type interaction with the non-collinear configuration of interacting waves in analogy to classical volume hologram. The memories based on the resonant interaction in $\Lambda_{-}^{-}$ schemes were extended to spatially-multimode case for the fast and adiabatic [9, 10] modes of operation. Typically, by the forward-propagating retrieval the single-pass schemes are less sensitive to diffraction and therefore are capable of achieving high density of storage of transverse spatial modes. An extensive numerical analysis [11] of parallel $\Lambda$-type memory capacity was performed in the paraxial approximation for a realistic shape of atomic ensemble.

The spatially resolving storage and retrieval of speckle-like patterns was demonstrated [12] experimentally, the orbital angular momentum modes were effectively stored and retrieved [13].

An important resource allowing for the manipulation of multiple quantized signals stored in quantum memories is provided by the use of classical control field with more complex spatial and temporal structure [14 [16] as compared to co- and counter-propagating illumination with quasi-monochromatic wave. The use of multi-atomic ensemble which occupies a significant part of the cavity volume, as opposed to variety of single-atom microcavitybased memories, makes it possible to achieve storage in multiple spatial modes of the collective spin and provides sensitivity to the control field propagation direction. By scanning the illumination angle [14] or under the condition of continuous phase-matching control across the control field beam [15] one can reproduce spatial and temporal structure of a weak signal pulse. The 2D addressability of degenerate cavity-assisted off-resonant memory by the illumination, close to orthogonal, allows for the retrieval on demand of the signals stored both in transverse and longitudinal collective spin waves [16].

In this paper we consider the operation mode of cavity-assisted off-resonant memory allowing not only to store and retrieve time sequences of light signals, but to perform an on-demand unitary "in-out" transformation of their quantized amplitudes. This mode of operation might be of interest as a step towards essential transformation of 
quantum states in the devices based on multivariate quantum memories. In this regime the memory is able to operate as a controllable delayed multi-port beamsplitter, capable of transformation of the delays, the durations and time shapes of signals in the sequence. In order to achieve this, we assume that longitudinal modes of the collective spin coherence of cold atomic ensemble are coherently controlled by the superpositions of pump waves propagating in the directions, close to orthogonal to the cavity axis. This is equivalent to the use of control field with the on-demand modulated transverse profile. First, by the write-in one can couple a time sequence of the incoming quantized signals to a given set of superpositions of orthogonal spin waves. By the readout, one can retrieve quantum states of the collective spin waves that are controllable superpositions of the initial ones and are on demand coupled to the output signal sequence.

We elaborate the theory of such light-matter interface for the spatially multivariate cavity-based off-resonant Raman-type quantum memory. Since in order to speed up the manipulation of sequences of many signals in multivariate memories it might be of interest to store relatively short light pulses of a given time shape, we also address the problem of optimal choice of the control field time profile for the cavity-based memory operation beyond the bad cavity limit.

\section{LIGHT-MATTER INTERACTION WITH SPATIALLY STRUCTURED CONTROL FIELD}

We consider memory scheme scheme based on a high-Q single-port ring cavity of length L, see Fig. 1. The $y$-polarized cavity field is

$$
E(\mathbf{r}, t)=i \sqrt{\frac{2 \pi \hbar \omega_{c}}{L}} a(t) \phi_{0}(x, y) \exp \left\{i\left(k_{c} z-\omega_{c} t\right\}+h . c .,\right.
$$

where $a(t)$ is the quantized cavity mode slow amplitude, that satisfies the standard commutation relation, $\left[a(t), a^{\dagger}(t)\right]=1$, and the transverse distribution of the field in $\{\mathrm{x}, \mathrm{y}\}$ plane is given by the normalized zero-order Hermite-Gauss profile $\phi_{0}(x, y)$,

$$
\int d x d y\left|\phi_{0}(x, y)\right|^{2}=1
$$

The empty cavity eigenfrequency is $\omega_{c}$, and $k_{c}=\omega_{c} / c$. The $y$-polarized input signal field, coupled to the cavity

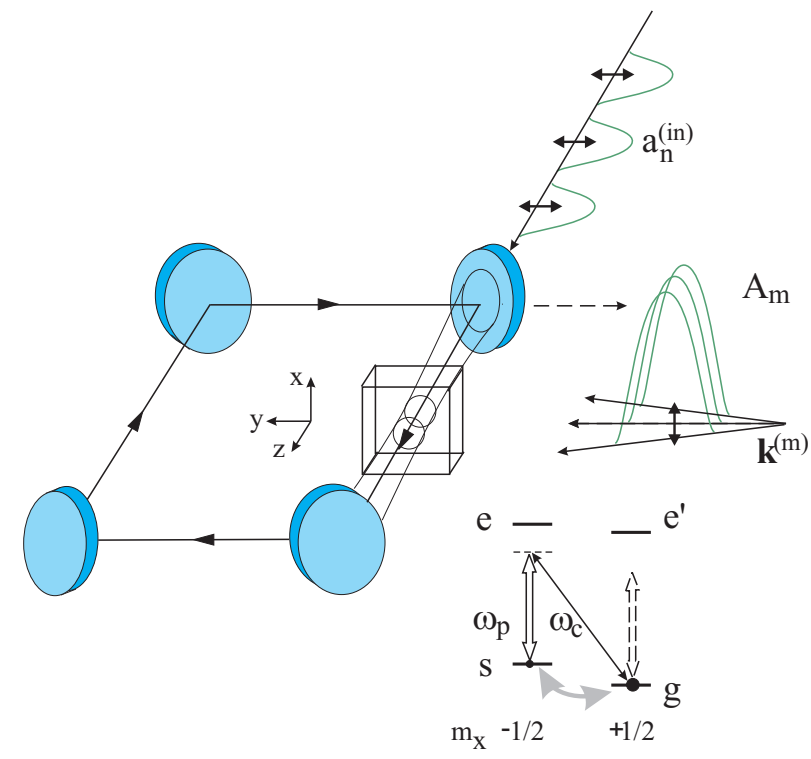

FIG. 1: Quantum memory scheme.

mode, at the input cavity mirror $(z=0)$ is

$$
E^{(i n)}(x, y, 0, t)=i \sqrt{\frac{2 \pi \hbar \omega_{c}}{c}} a^{(i n)}(t) \phi_{0}(x, y) \exp \left\{-i \omega_{c} t\right\}+h . c .
$$

with the commutation relation for the input field slow amplitude of the form $\left[a^{(i n)}(t), a^{(i n) \dagger}\left(t^{\prime}\right)\right]=\delta\left(t-t^{\prime}\right)$. 
The quantized field evolution in presence of the decay at $C / 2$ rate and of the field $a^{(i n)}(t)$ at the input port is described by

$$
\dot{a}(t)=-\frac{C}{2} a(t)+\sqrt{C} a^{(i n)}(t), \quad a^{(o u t)}(t)=\sqrt{C} a(t)-a^{(i n)}(t) .
$$

A spatially distributed ensemble of motionless atoms with angular momentum $1 / 2$ both in the ground and in the excited states is located inside the cavity, see Fig. 1. We assume for simplicity that transverse dimensions of the interaction area are determined by the cavity field waste, and the medium length is $L_{z}$. The ground state collective spin coherence amplitude is given by

$$
b(\mathbf{r}, t)=\frac{J_{y}(\mathbf{r}, t)+i J_{z}(\mathbf{r}, t)}{\sqrt{2 n_{a}\left\langle J_{x}^{a}\right\rangle}},
$$

where $J_{i}(\mathbf{r}, t)$ for $i=x, y, z$ are the spin projection operators, and the summation over atoms with the concentration $n_{a}$ is performed,

$$
J_{i}(\mathbf{r}, t)=\sum_{a} J_{i}^{a}(t) \delta\left(\mathbf{r}-\mathbf{r}_{a}\right) .
$$

The quantum memory regime implies that the number of atoms in the initial state $J_{x}^{a}=+1 / 2$ remains almost unchanged. This allows one to substitute the operator $J_{x}^{a}$ with its mean value $\left\langle J_{x}^{a}\right\rangle$, and to arrive at the averaged over random positions of atoms bosonic commutation relation of the form

$$
{\overline{\left[b(\mathbf{r}, t), b^{\dagger}\left(\mathbf{r}^{\prime}, t\right)\right.}}^{a}=\delta\left(\mathbf{r}-\mathbf{r}^{\prime}\right) .
$$

Due to the presence of constant magnetic field, oriented along $x$-direction, the collective spin rotates at frequency $\Omega=\omega_{s g}$,

$$
\dot{b}(\mathbf{r}, t)=-i \Omega b(\mathbf{r}, t) .
$$

Extending the addressable memory configuration of [16], we assume the pump (control) field to be a controlled superposition of strong classical $x$-polarized plane waves at frequency $\omega_{p}$, propagating in directions in the $\{y, z\}$ plane, close to orthogonal to the system axis $z$. Under the condition of the Raman resonance, $\omega_{c} \approx \omega_{p}+\Omega$, and for large enough magnetic splitting, $\Omega \gg C / 2$, the cavity maintains quantized field in the memory channel, see Fig. 1. and suppresses the entanglement channel. The last one is off-resonant to the cavity eigenfrequency.

Assuming a large frequency detuning from the electronic transition, $\left|\omega_{c}-\omega_{e g}\right| \gg \Omega, C$, one can apply the effective QND Hamiltonian [1], that describes the light-matter interaction:

$$
H_{e f f}=\frac{2 \pi \omega_{c}|d|^{2}}{\left(\omega_{c}-\omega_{e g}\right) L} \int d \mathbf{r} J_{z}(\mathbf{r}, t) S_{z}(\mathbf{r}, t),
$$

where $d$ is dipole matrix element, $\omega_{e g}$ is the transition frequency, $S_{z}(\mathbf{r}, t)=\left[A_{r}^{\dagger}(\mathbf{r}, t) A_{r}(\mathbf{r}, t)-A_{l}^{\dagger}(\mathbf{r}, t) A_{l}(\mathbf{r}, t)\right]$ is $z$-projection of the Stokes vector. Here $A_{r, l}$ are slow amplitudes of the right and left polarized fields rotating in the $(x, y)$ plane, such that $E_{r, l}(\mathbf{r}, t)=i\left(2 \pi \hbar \omega_{c} / L\right)^{1 / 2} A_{r, l}(\mathbf{r}, t)+h . c$. The pump field is composed of $\mathrm{N}$ plane waves with the amplitudes $A_{m}(t), m=1 \ldots N$, propagating in different directions in the $(y, z)$ plane. Hence, the atoms are interacting with the field

$$
A(\mathbf{r}, t)=\sum_{m=1}^{N} A_{m}(t) \exp \left\{i\left(k_{z}^{(m)} z+k_{y}^{(m)} y-\omega_{p} t\right)\right\} \mathbf{e}_{x}+a(t) \phi_{0}(x, y) \exp \left\{i\left(k_{c} z-\omega_{c} t\right\} \mathbf{e}_{y} .\right.
$$

Here $k_{z}^{(m)}$ and $k_{u}^{(m)}$ are the wave vector projections for $m^{\prime}$ th pump wave. Following the procedure described in more detail in [16, 22], we retain the bilinear in $A_{m}(t)$ and $a(t)$ contributions to $H_{e f f}$, and find the terms that come to the evolution equations (44 (7) in the Heisenberg picture due to light-matter interaction. The contributions related to the entanglement generation channel $\left(\dot{a} \sim b^{\dagger}, \dot{b} \sim a^{\dagger}\right)$ are oscillating at frequency $\pm 2 \Omega$ and are averaged out on the time scale of interest $1 / C$.

Note that we do not account here for atomic relaxation. There are also omitted contributions to the effective QND Hamiltonian that give rise to the cavity frequency correction due to the matter refraction index, and to the atomic levels light shifts induced by the control field. In what follows we imply that $\omega_{c}$ is the corrected cavity frequency, and the common time dependent light shifts of the ground state sublevels mutually compensate.

The cavity and the collective spin evolution equations are then found to be

$$
\dot{a}(t)=-\frac{C}{2} a(t)-
$$




$$
\begin{gathered}
i Q \int d \mathbf{r} b(\mathbf{r}, t) \sum_{m=1}^{N} A_{m}(t) \phi_{0}^{*}(x, y) \exp \left\{i\left[\left(k_{z}^{(m)}-k_{c}\right) z+k_{y}^{(m)} y+\Omega t\right]\right\}+\sqrt{C} a^{(i n)}(t), \\
\dot{b}(\mathbf{r}, t)=-i \Omega b(\mathbf{r}, t)-i Q \sum_{m=1}^{N} a(t) A_{m}^{*}(t) \phi_{0}(x, y) \exp \left\{-i\left[\left(k_{z}^{(m)}-k_{c}\right) z+k_{y}^{(m)} y+\Omega t\right]\right\},
\end{gathered}
$$

where

$$
Q=\frac{\pi \omega_{c}|d|^{2}}{\hbar\left(\omega_{c}-\omega_{e g}\right) L} \sqrt{2 n_{a}\left\langle J_{x}^{a}\right\rangle}
$$

The evolution equations above describe the cavity field interaction with a set of independent collective spin waves, controlled by the pump components, given the space profiles that appear in (9, 10) can be considered as mutually orthogonal. To be specific, we assume that atomic distribution is uniform within the cavity field waste, and $L_{z}$ is the medium dimension along $z$-axis. The factors $\exp \left\{i\left[\left(k_{z}^{(m)}-k_{c}\right) z\right\}\right.$ in (9) provide the orthogonality given the $z$-projections of the pump wave vectors are chosen in such a way that $\left(k_{z}^{(n)}-k_{z}^{(m)}\right)=2 \pi(n-m) / L_{z}$. For the pump waves propagating in the directions close to $y$, when $\left|k_{y}^{(m)}\right| \approx\left|k^{(m)}\right|=\omega_{p} / c \gg\left|k_{z}^{(m)}\right|$, the factor $\exp \left(i k_{y}^{(m)} y\right)$ can be substituted by $\exp \left[i\left(\omega_{p} / c\right) y\right]$ within the cavity field waste, and the transverse profile of the spin waves involved into interaction is given by $\phi_{0}(x, y) \exp \left[i\left(\omega_{p} / c\right) y\right]$.

We arrive at the following decomposition of the collective spin amplitude,

$$
b(\mathbf{r}, t)=\sum_{m=1}^{N} b_{m}(t) \psi_{m}(\mathbf{r}) e^{-i \Omega t}+\ldots,
$$

where $\psi_{m}(\mathbf{r})$ are orthogonal spatial profiles of the spin waves interacting with the cavity field,

$$
\psi_{m}(\mathbf{r})=\frac{1}{\sqrt{L_{z}}} \phi_{0}(x, y) \exp \left\{-i\left[\left(k_{z}^{(m)}-k_{c}\right) z+\left(\omega_{p} / c\right) y\right\},\right.
$$

and "..." stands for contribution of all other degrees of freedom of the collective spin. The commutation relation (6) yields $\left[b_{n}(t), b_{m}^{\dagger}(t)\right]=\delta_{n m}$. Inserting the decomposition (11) into (9, 10), we reduce the evolution equations to the form

$$
\begin{gathered}
\dot{a}(t)=-\frac{C}{2} a(t)+\sqrt{C} a^{(i n)}(t)-i \sum_{m=1}^{N} k_{m}(t) b_{m}(t), \\
\dot{b}_{m}(t)=-i k_{m}^{*}(t) a(t) .
\end{gathered}
$$

The coupling parameter $k_{m}(t)=Q \sqrt{L_{z}} A_{m}(t)$ gives the state exchange frequency between the $m$ 'th spin wave and the cavity field.

Consider time sequence of $N$ non-overlapping quantized input signals of duration $\Delta T^{(W)}$ with the amplitudes $a_{n}^{(i n)}$, such that $\left[a_{n}^{(i n)}, a_{m}^{(i n) \dagger}\right]=\delta_{n m}$, where $\left\{t_{n}\right\}$ are the initial time moments of the signals,

$$
a^{(i n)}(t)=\sum_{n=1}^{N} a_{n}^{(i n)} f\left(t-t_{n}\right)+\ldots, \quad \int_{\Delta T^{(W)}} d t|f(t)|^{2}=1,
$$

where $f\left(t-t_{n}\right)$ are normalized time profiles of the temporal modes to be written, and "..." stands for the contribution of other temporal modes that ensure correct commutation relations.

For the write-in of a single input signal $\sim a^{(i n)} f(t)$ onto a single matter oscillator on the $\left(0, T^{(W)}\right)$ time interval, the Heisenberg-Langevin equations above yield the following "in-out" relation,

$$
b\left(T^{(W)}\right)=K^{(W)} a^{(i n)}+\sqrt{1-\left|K^{(W)}\right|^{2}} v^{(i n)},
$$

where the write-in efficiency is $\eta^{(W)}=\left|K^{(W)}\right|^{2} \leq 1$, the bosonic amplitude $v^{(i n)}$ accounts for the initial vacuum contributions of the cavity field, the spin wave, and other input temporal modes that are present by the $T^{(W)}$ time moment. The problem of the optimal coupling parameter $k(t)$ control in order to achieve high efficiency for the signal temporal mode of interest was thoroughly discussed in literature, in what follows we shall address some aspects of such control beyond the bad cavity limit. 


\section{MEMORY AS MULTI-PORT BEAMSPLITTER}

Assume that for the write-in of the n'th signal from the sequence, the coupling parameters are taken in the form $k_{m}(t)=U_{n m}^{(W)} k\left(t-t_{n}\right)$, where $U^{(W)}$ is an arbitrary unitary matrix. Inserting this to (12, 13), one finds that the evolution equations reduce to the case of interaction with a single spin oscillator with the amplitude

$b_{n}^{\prime}(t)=\sum_{m} U_{n m}^{(W)} b_{m}(t)$. Since before writing the signal sequence, the collective spin is supposed to be in a vacuum state, the same is true for all amplitudes $b_{n}^{\prime}$. We also assume that between the write-in cycles the cavity field restores its initial vacuum state due to finite cavity lifetime. That is, we can apply the transformation (15) incrementally for $n=1 \ldots N$ in the following form:

$$
b_{n}^{\prime}\left(t_{n}+T^{(W)}\right)=K^{(W)} a_{n}^{(i n)}+\sqrt{1-\left|K^{(W)}\right|^{2}} v_{n}^{(i n)},
$$

where the unitary transform ensures that $\left[b_{n}^{\prime}(t), b_{m}^{\prime \dagger}(t)\right]=\delta_{n m}$. To summarize, by the write-in of a time sequence of quantized input signals, one can record their quantum state onto controllable superpositions of the collective spin waves. In the initial basis for matter variables this yields,

$$
b_{n}\left(t_{n}+T^{(W)}\right) \sim K^{(W)} \sum_{m}\left(U^{(W) \dagger}\right)_{n m} a_{m}^{(i n)} .
$$

Here and in what follows vacuum terms are omitted for brevity.

For the readout stage we assume representation of the output field similar to (14), with the time duration $\Delta T^{(R)}$ of a retrieved signal from the sequence, and its time profile $g\left(t-t_{n}\right)$. For the retrieval of a single output signal $\sim a^{(o u t)} g(t)$ from a single matter oscillator on the $\left(0, T^{(R)}\right)$ time interval, the evolution equations above are solved to obtain $a^{(\text {out })} \sim K^{(R)} b(0)$.

It is assumed that the time shape of the coupling parameter $k(t)$ during the readout ensures efficient retrieval of the temporal mode $g(t)$. For the n'th signal from the output sequence, the coupling parameters are chosen to be $k_{m}(t)=U_{n m}^{(R)} k\left(t-t_{n}\right)$, where $U^{(R)}$ is unitary matrix. As before, the evolution equations reduce to the case of interaction with a single spin oscillator with the amplitude $b_{n}^{\prime \prime}(t)=\sum_{m} U_{n m}^{(R)} b_{m}(t)$. The collective spin is supposed to be in the state, prepared during the write-in, and the state of incoming quantized field is vacuum. We also assume that between the readout cycles the cavity field restores its initial vacuum state due to finite cavity lifetime. We arrive at

$$
a_{n}^{(\text {out })} \sim K^{(R)} b_{n}^{\prime \prime}\left(t_{n}\right)=K^{(R)} \sum_{m} U_{n m}^{(R)} b_{m}\left(t_{n}\right) .
$$

The complete "in-out" transformation of the input sequence of quantized signals to the output one is composed of (17) and (18), and its essential part (apart from the vacuum contributions) is found to be

$$
a_{n}^{(\text {out })} \sim K^{(R)} K^{(W)} \sum_{m} U_{n m}^{(R W)} a_{m}^{(i n)},
$$

where $U^{(R W)}=U^{(R)} U^{(W) \dagger}$. For $U^{(R)}=U^{(W)}$, when $U^{(R W)}=I$, this yields $a_{n}^{(\text {out })}=a_{n}^{(\text {in) }}$, but even in this simplest case the memory is able to transform the delays, the durations, and time shapes of the signals in the sequence, matching needs of next stage of the signals processing. Given each row of $U^{(R W)}$ contains a unit in an arbitrary position, the memory will change the signals order between the input and the output. In general case, the described multivariate mode of operation makes it possible to retrieve on demand superpositions of the input amplitudes. For instance, if the complete transformation matrix $U^{(R W)}$ corresponds to $1: 1$ beamsplitter $(N=2)$, an input sequence of two signals, squeezed in orthogonal quadratures, is retrieved in the form of two entangled pulses. For $N>2$ one can simulate a multi-slit interference of arbitrarily delayed and transposed signals with losses limited by the memory efficiency.

As for experimental aspects of the proposed scheme, the effective shape of an ultracold atomic ensemble may differ from the simple model we have used in order to introduce orthogonal collective spin waves. For Gaussian distribution of atoms along the system axis, there are more complex profiles of the pump field, as compared to the plane waves with discrete values of the wave vector z-component $k_{i z}$, that interact with orthogonal matter waves. As a suitable solution, one could use plane pump waves with larger increment of $k_{i z}$. This would result in smaller overlap of the relevant spin waves, in analogy to partially overlapping Glauber coherent states. More detailed discussion of this issue is beyond the scope of this paper.

In order to effectively excite a high-quality cavity with active medium inside, one has to minimize reflection of the input signal during the write-in. This can be achieved by the impedance matching, that is, by matching of the signal absorption by the medium placed inside the cavity, with the cavity losses. It was demonstrated theoretically, that this approach works perfectly in the bad cavity limit for the atomic spin [17], the atomic frequency comb [18], and the controlled reversible inhomogeneous broadening [19] memories. 
Recent experimental demonstration [20] of the cavity-assisted cold atom memory with quasi-Gaussian control field pulses was interpreted in terms of adiabatic elimination of more general form [21].

In the regime of multi-signal sequences, like in our proposal, it might be of interest to speed up the manipulation of an elementary input signal beyond the bad cavity regime in order to come to a suitable compromise between the speed and the efficiency, which is limited also by other imperfections of the scheme. One can achieve good efficiencies by matching the time shape of the light-matter coupling $k(t)$ with an on-demand smooth temporal mode of the signal $f(t)$ (by the write-in), or $g(t)$ (by the readout) for the durations of the order of few cavity lifetimes [22, 23]. It is worth noticing that the impedance matching approach allows to find a set of the light-matter coupling parameter time profiles, that correspond to almost equal values of the efficiency [23], as illustrated in fig. 2] Having in mind the readout, in order to achieve a desired time shape of the signal, it might be needed to transfer some cavity excitations back to collective spin. Such transfer occurs possible for a set of the control field and the collective spin amplitudes, given their relative phase ensures absorption of the excess cavity photons.

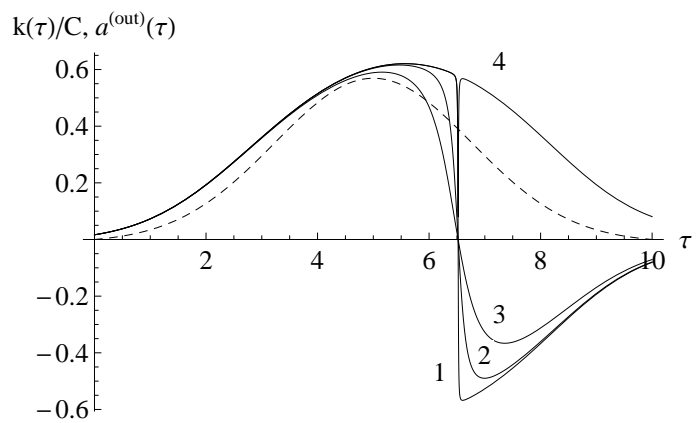

FIG. 2: Plots of (dashed line) normalized Gaussian output signal $a^{(\text {out })}(\tau)$ and (solid lines) several corresponding dimensionless coupling parameters $k(\tau) / C$ vs. time $\tau=C t$ (that is, time in units of the cavity lifetime). Here $T^{(R)}=10 / C$, the readout efficiencies are $\eta^{(R)}=(1,4) 0.958$, (2) 0.957 , and (3) 0.946, respectively.

\section{CONCLUSION}

We have presented a detailed theory of the cavity assisted quantum memory with many longitudinal modes of the collective spin coherence, controlled by the spatially structured classical pump field. We have demonstrated that by the write-in in this regime of operation, the memory is able to couple a time sequence of the incoming quantized signals to a given set of superpositions of orthogonal spin waves. By the readout, one can retrieve quantum states of the collective spin waves that are controllable superpositions of the initial ones and are on demand coupled to the output signal sequence. The memory operation in the regime of controllable delayed multi-port beamsplitter, capable of transformation of the delays, the durations and time shapes of signals in the sequence, might be of interest for various tasks of quantum information.

This work was supported by the Russian Foundation for Basic Research through the Projects 13-02-00254-a and 15-02-03656-a. One of the authors (A. V.) acknowledges support by the Government of St. Petersburg grant for young researches.

[1] K. Hammerer, A. S. Sorensen, and E. S. Polzik, Rev. Mod. Phys. 82, 1041 (2010).

[2] F. Bussieres, N. Sangouard, M. Afzelius, H. de Riedmatten, C. Simon and W. Tittel, J. Mod. Optics 60 (18), 1519 (2013).

[3] New J. Phys. 15 (2013), special issue on quantum memories.

[4] D. V. Vasilyev, I. V. Sokolov, E. S. Polzik, Phys. Rev. A 77, 020302(R) (2008).

[5] K. Tordrup, A. Negretti, and K. Moelmer, Phys. Rev. Lett. 101, 040501 (2008).

[6] K. Surmacz, J. Nunn, K. Reim, K. C. Lee, V. O. Lorenz, B. Sussman, I. A. Walmsley, D. Jaksch, Phys. Rev. A 78, 033806 (2008).

[7] D. V. Vasilyev, I. V. Sokolov, and E. S. Polzik, Phys. Rev. A 81, 020302 (2010).

[8] D. V. Vasilyev and I. V. Sokolov, Eur. Phys. J. D 66, 294 (2012).

[9] T. Golubeva, Yu. M. Golubev, O. Mishina, A. Bramati, J. Laurat, E. Giacobino, Eur. Phys. J. D 66, 275 (2012).

[10] K. Tikhonov, K. Samburskaya, T. Golubeva, Yu. Golubev, Phys. Rev. A 89, 013811 (2014).

[11] E. Zeuthen, A. Grodecka-Grad, and A. S. Sørensen, Phys. Rev. A 84, 043838 (2011).

[12] R. Chrapkiewicz, and W. Wasilewski, Optics Express 20 (28), 29540 (2012). 
[13] A. Nicolas, L. Veissier, L. Giner, E. Giacobino, D. Maxein, and J. Laurat, Nature Phot., 8, 234 (2014).

[14] X. Zhang, A. Kalachev, and O. Kocharovskaya, Phys. Rev. A 87, 013811 (2013).

[15] A. Kalachev, and O. Kocharovskaya, Phys. Rev. A 88, 033846 (2013).

[16] A. N. Vetlugin and I. V. Sokolov, Eur. Phys. J. D 68, 269 (2014).

[17] A. V. Gorshkov, A. Andre, M. D. Lukin, and A. S. Sorensen, Phys. Rev. A 76, 033804 (2007).

[18] M. Afzelius, and C. Simon, Phys. Rev. A 82, 022310 (2010).

[19] S. A. Moiseev, S. N. Andrianov, and F. F. Gubaidullin, Phys. Rev. A 82, 022311 (2010).

[20] E. Bimbard, R. Boddeda, N. Vitrant, A. Grankin, V. Parigi, J. Stanojevic, A. Ourjoumtsev, and P. Grangier, Phys. Rev. Lett. 112, 033601 (2014).

[21] J. Stanojevic, V. Parigi, E. Bimbard, R. Tualle-Brouri, A. Ourjoumtsev, and P. Grangier, Phys. Rev. A 84, 053830 (2011).

[22] A. N. Vetlugin and I. V. Sokolov, Opt. Spectr. 115(6), 980 (2013).

[23] V. V. Kuzmin, A. N. Vetlugin and I. V. Sokolov, Opt. Spectr. 119(6), 1004 (2015). 